\title{
Dengue Infection Induced Diffuse Alveolar Hemorrhage
}

\author{
Vishal Singh ${ }^{1}$, Satish Chandra Mishra ${ }^{2}$, Pulkit Singh ${ }^{3}$, Pradeepa AM ${ }^{4}$ \\ ${ }^{1}$ Division of Nephrology, Department of Medicine, 7 AF Hospital, Kanpur, Uttar Pradesh, India; ${ }^{2}$ Department of Interventional \\ Cardiology, Military Hospital (CTC), Pune, Maharashtra, India; ${ }^{3}$ MS Ramaiah Medical College, Bangalore, Karnataka, India; \\ ${ }^{4}$ Department of Medicine, 7 AF Hospital, Kanpur, Uttar Pradesh, India.
}

\section{Corresponding Author: \\ Dr Vishal Singh \\ Email: vishal23415@gmail.com}

This is an Open Access article distributed under the terms of the Creative Commons Attribution License (creativecommons.org/ licenses/by/3.0).

Received Accepted Published

December 26, 2019

March 26, 2020

April 5, 2020

\begin{abstract}
Background: Dengue fever is a commonly encountered arthropod-borne viral disease. The spectrum of the disease varies widely, and at times it may present with unusual complications. One such dreaded but rare complication is diffuse alveolar hemorrhage (DAH). Case Report: A 42 years old lady having no co-morbidities presented with dengue infection. The presentation included fever, gum bleed, and asthenia. The examination showed low oxygen saturation and clear chest. The discordance between clinical findings and the oxygen saturation led to a cascade of investigations, culminating in the diagnosis of DAH. She was managed symptomatically, with full recovery. Conclusion: DAH is an uncommon, life-threatening, complication of dengue infection. A high index of suspicion, prompt evaluation and early institution of appropriate therapy hold the key to successfully manage such patients.
\end{abstract}

Keywords: Arbovirus Infections, Dengue, Fever, Hemorrhage, Oxygen.

\section{Introduction}

Dengue infection has emerged as a major public health concern worldwide. It is an acute infectious disease caused by four distinct serotypes of the dengue virus (DENV 1-4). It is transmitted by the bite of a female mosquito of genus Aedes. In humans, it causes dengue fever, dengue hemorrhagic fever (DHF) and dengue shock syndrome [1]. The spectrum of the disease varies widely, from asymptomatic to life-threatening. Over the last few decades, not only has there been a global resurgence of the disease but the infection has spread to involve new geographical areas [2]. In rapidly urbanizing, densely populated, tropical countries like India, with limited health care resources this suggests an ominous future.

The majority of the patients with dengue infection require only symptomatic therapy and reassurance, a small percentage however develops complications necessitating hospitalization. The commonly encountered complications include bleeding, shock, organ dysfunction and metabolic acidosis. The involvement of the lower respiratory tract is uncommon. We present the case of a young lady with DENV induced diffuse alveolar hemorrhage (DAH).

\section{Case Report}

The patient, a 42 years old lady had been well until four days prior to admission when she developed high-grade fever, gum bleed, headache, and asthenia. She appeared anxious but well preserved and was noted to be dyspneic while walking towards the examination room. The examination showed a pulse of 98 per minute, blood pressure of 102/64 $\mathrm{mm} \mathrm{Hg}$, respiratory rate of $28 / \mathrm{min}$ and the oxygen saturation at room air was $74 \%$. Non-blanching confluent rash, involving the abdomen and the back was noted. The auscultation of the chest was normal and the abdomen was soft. There was no ongoing bleed from any site including the oral 
cavity. The investigations showed, hemoglobin: $10.4 \mathrm{~g} / \mathrm{dL}$, total leucocyte count: $2400 / \mathrm{mm}^{3}$, platelet count: $0.35 \mathrm{lac} / \mathrm{mm}^{3}$, and mildly elevated liver aminotransferases. The other biochemical parameters were normal. There was an ongoing dengue epidemic in the region, and hence she was evaluated for the same. The dengue serology for NS1 antigen, using both rapid diagnostic test kit and enzyme-linked immunosorbent assay-based antigen detection test was positive. She was diagnosed as a case of dengue infection.

In view of the discordance between the oxygen saturation and the clinical findings, further investigations were performed. The chest radiograph showed bilateral airspace consolidation [Fig.1]. A high-resolution computed tomography of the lungs showed bilateral alveolar air space consolidation, ground glass-opacities and pleural effusion [Fig. 2]. With a strong suspicion of DAH, a bronchoscopy was performed; the bronchoalveolar lavage was blood-tinged. Other causes of DAH were ruled out based on the history, clinical examination, absence of anti-nuclear antibodies and anti-neutrophilic cytoplasmic antibodies and the presence of normal coagulation profile and complement factors.

She was managed with single donor platelet transfusion, antipyretics, oxygen supplementation, and intravenous fluid. By day 3 of hospitalization, the fever subsided and the oxygen saturation normalized. A repeat chest radiograph done a few days later, showed spontaneous clearance of the pulmonary opacities [Fig. 3].

\section{Discussion}

DENV is the commonest human arbovirus infection worldwide. After the incubation period, the disease begins abruptly and passes through three phases: febrile, critical and recovery phase. The severe forms of dengue are characterized by plasma leakage, thrombocytopenia, and hemorrhage. At times, dengue infection may result

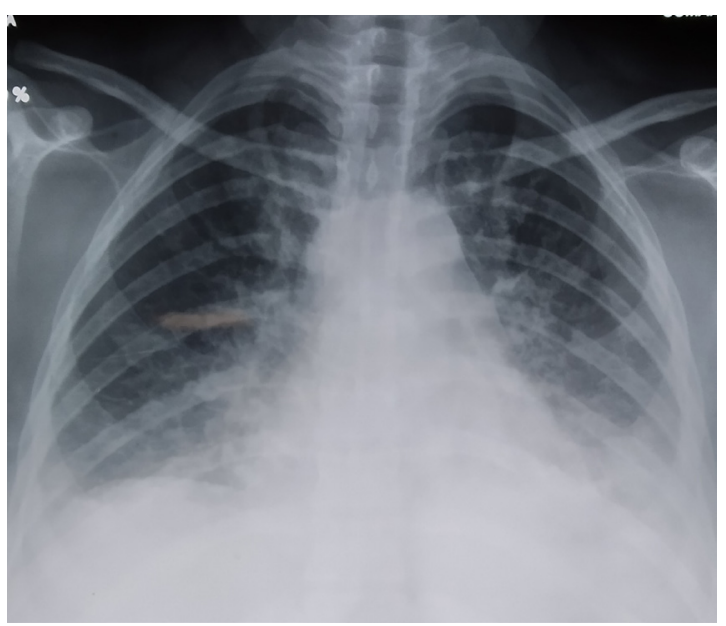

Fig.1: Chest radiograph showing bilateral airspace consolidation.

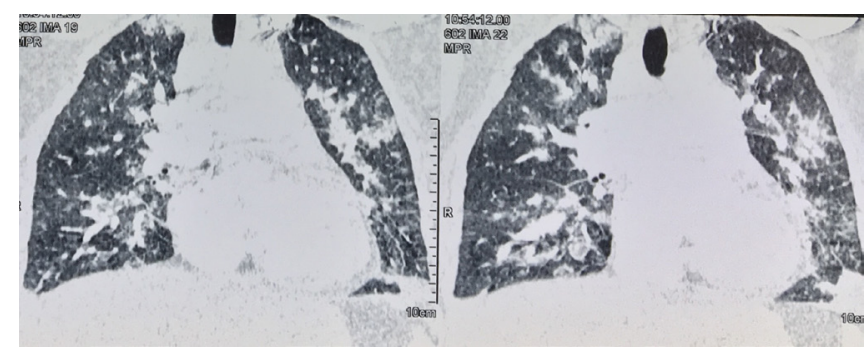

Fig.2: Non-contrast computed tomography of the lungs showing bilateral alveolar air space consolidation and ground glass-opacities.

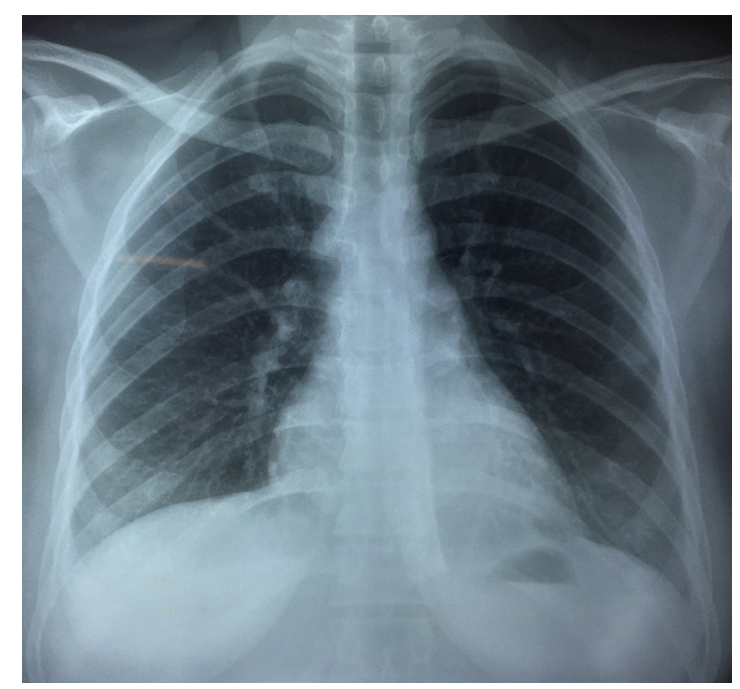

Fig.3: A repeat chest radiograph showing spontaneous clearance of the pulmonary opacities. 
in organ dysfunction. While dengue hepatitis is relatively common, others like myocarditis, acute tubular injury, and encephalitis are less common. Occasional patients may have lower respiratory tract involvement; hemoptysis occurs in $1.4 \%$ of dengue infection [3].

$\mathrm{DAH}$ is a potentially life-threatening disorder, caused by pulmonary capillaritis. It commonly presents with hemoptysis, anemia and diffuse alveolar infiltrates. The commonest etiology for DAH is autoimmune disorders. Association with dengue infection is rare, and the first case was described in 1993 [4]. Since then, occasional similar cases have been reported [5-8]. Our patient differed significantly from the existing sparse case reports. She was well preserved, did not present with the classical symptom i.e. hemoptysis, and the clinical outcome with conservative management was favorable. The presentation was so subtle that had it not been for a mismatch between the clinical findings and oxygen saturation, we would have never suspected and evaluated her for DAH. Looking objectively, the subtle signs which prompted us to perform a chest radiograph, which in turn led to the diagnosis of DAH is often ignored in day to day clinical practice, which makes us feel that the condition often remains unrecognized and undiagnosed. The biological cascade, leading to DAH in dengue fever is not known. We feel the cause is multifactorial with thrombocytopenia, platelet dysfunction, coagulopathy, altered alveolarcapillary membrane permeability and direct injury to the alveolar lining cells by DENV all playing a vital and interlinking role in the pathogenesis.

The management of patients with DHF remains supportive. The role of prophylactic platelet transfusion remains controversial. Some studies have shown that platelet transfusion does not prevent the development of severe bleeding or shorten the time to bleeding cessation. Studies have also shown that the improvement in platelet count, following a platelet transfusion, is transient, lasting few hours [9]. We feel that the decision to transfuse platelets should be based on the overall clinical context, rather than any arbitrary cutoff. Our patient had DAH, and peculiar site of bleed precluded us from real-time assessment, whether the bleed is ongoing or has stopped. In view of this, despite the platelet count still being more than $10,000 / \mathrm{mm}^{3}$, we transfused her with single donor platelets. Notwithstanding the controversies, the cornerstone of treatment remains meticulous maintenance of fluid and electrolyte balance along with supportive care. The mortality rate, with appropriate intensive supportive care, may be reduced to less than $1 \%$ [10]. It is hence imperative, that in the appropriate setting, while dealing with a patient of DAH, DHF should be considered one of the differentials.

\section{Conclusion}

DENV infection has a wide spectrum of presentations. Rarely, it may present with DAH. A high index of suspicion, prompt evaluation, and appropriate therapy hold the key to the successful management of these patients.

Contributors: VS, SCM, PS and PAM did literature search and patient management. VS and PS drafted the manuscript. All authors have approved the final version of the manuscript. VS will act as a study guarantor, and is responsible for all aspects of the study.

Funding: None; Competing interests: None stated.

\section{References}

1. Balmaseda A, Hammond SN, Pe'rez L, Tellez Y, Saborı'o SI, Mercado JC, et al. Serotype-specific differences in clinical manifestations of dengue. Am J Trop Med Hyg. 2006;74:449-456.

2. Kyle JL, Harris E. Global spread and persistence of dengue. Annu Rev Microbiol. 2008;62:71-92.

3. Hayes GC, Manaloto CR, Gonzales A, Ranoa CP. Dengue infections in the Philippines: clinical and virological findings in 517 hospitalized patients. Am J Trop Med Hyg. 1988;39:110-116.

4. Liam CK, Yap BH, Lam SK. Dengue fever complicated by pulmonary haemorrhage manifesting as haemoptysis. J Trop Med Hyg. 1993;96:197-200.

5. Setlik RF, Ouellette D, Morgan J, McAllister CK, Dorsey $\mathrm{D}$, Agan BK, et al. Pulmonary hemorrhage syndrome associated with an autochthonous case of dengue hemorrhagic fever. South Med J. 2004;97:688-691. 
6. Sharma SK, Gupta BS, Devpura G, Agarwal A, Anand S. Pulmonary haemorrhage syndrome associated with dengue haemorrhagic fever. J Assoc Physicians India. 2007;55:729-730.

7. Ralapanawa DMPUK, Jayawickreme KP, Ekanayake EMM, Jayalath T, Herath D. Fatal massive pulmonary hemorrhage in dengue infection. Epidemiology (Sunnyvale). 2016;6:251.

8. Marchiori E, Ferreira JL, Bittencourt CN, de Arau' joNeto
CA, Zanetti G, Mano CM, et al. Pulmonary hemorrhage syndrome associated with dengue fever, high-resolution computed tomography findings: a case report. Orphanet J Rare Dis. 2009;4:8.

9. Lum LC, Abdel-Latif Mel-A, Goh AY, Chan PW, Lam SK. Preventive transfusion in dengue shock syndrome-is it necessary? J Pediatr. 2003;143:682-684.

10. Chaturvedi UC. The curse of dengue. Indian J Med Res. 2006;124:467-470. 